International Journal of Pure and Applied Mathematics

Volume 111 No. 2 2016, 165-178

ISSN: 1311-8080 (printed version); ISSN: 1314-3395 (on-line version)

url: http://www.ijpam.eu

doi: 10.12732 /ijpam.v111i2.3

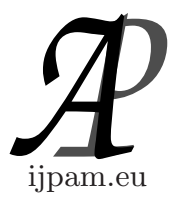

\title{
A FOUR-STAGE THIRD-ORDER SYMPLECTIC EXPLICIT TRIGONOMETRICALLY-FITTED RUNGE-KUTTA-NYSTRÖM METHOD FOR THE NUMERICAL INTEGRATION OF OSCILLATORY INITIAL-VALUE PROBLEMS
}

\author{
M.A. Demba ${ }^{1}$, N. Senu ${ }^{2}$, F. Ismail ${ }^{3}$ \\ ${ }^{1}$ Department of Mathematics \\ Universiti Putra Malaysia \\ 43400 UPM Serdang, Selangor, MALAYSIA \\ ${ }^{2,3}$ Department of Mathematics \\ and Institute for Mathematical Research \\ Universiti Putra Malaysia \\ 43400 UPM Serdang, Selangor, MALAYSIA
}

\begin{abstract}
In this work, a third-order four-stage symplectic explicit trigonometrically-fitted Runge-Kutta-Nyström (RKN) method for the numerical integration of second order initial value problems with oscillatory solutions based on Simos technique is constructed. The numerical results obtain signify the accuracy of the proposed method in comparison with other symplectic and non-symplectic RKN methods.
\end{abstract}

AMS Subject Classification: 65L05, 65L06

Key Words: trigonometric fitting, RKN methods, periodic initial value problems

Received: $\quad$ May 22, 2016

Revised: $\quad$ November 12, 2016

Published: $\quad$ December 11, 2016

$\S_{\text {Correspondence author }}$
(C) 2016 Academic Publications, Ltd. url: www.acadpubl.eu 


\section{Introduction}

In this paper, we consider the construction of a symplectic explicit trigonometrically-fitted Runge-Kutta-Nyström (RKN) method for the numerical integration of oscillatory initial value problems of the form

$$
\begin{gathered}
y^{\prime \prime}=f(x, y), \quad x \in\left[x_{0}, X\right], \\
y\left(x_{0}\right)=y_{0}, \quad y^{\prime}\left(x_{0}\right)=y_{0}^{\prime},
\end{gathered}
$$

whose first derivative does not appear explicitly. These oscillatory problems often arise in different fields of applied sciences such as: astrophysics, chemistry, molecular dynamics, celestial mechanics, theoretical physics, physical chemistry, nuclear physics and electronics. Van de Vyver in $[1,2,3]$ proposed a symplectic exponentially fitted modified Runge-Kutta-Nyström method for the numerical integration of orbital problems, a fourth-order symplectic exponentially fitted integrator and a symplectic Runge-Kutta-Nyström method with minimal phase-lag, Tocino and Vigo-Aguiar in [4] proposed symplectic conditions for exponential fitting Runge-Kutta-Nyström methods. Recently, Kalogiratou et al in [5] proposed a fourth order modified trigonometrically fitted symplectic Runge-Kutta-Nyström method. More recently, Franco and Gomez in [6] proposed symplectic explicit methods of Runge-Kutta-Nyström type for solving perturbed oscillators. Symplectic numerical methods are widely used for solving Hamiltonian systems; which preserves the symplectic structure in phase space. Hamiltonian systems are systems of first order ODEs that can be expressed as:

$$
\frac{d y}{d t}=-\frac{\partial H}{\partial x}, \quad \frac{d x}{d t}=\frac{\partial H}{\partial y}
$$

where $x, y \in \Re^{d}$ and $\mathrm{H}$ is a twice continuously differentiable function $H$ : $U \rightarrow \Re^{2 d}\left(U \subset \Re^{2 d}\right.$ is an open set). Symplecticity is a distinguishing feature of Hamiltonian systems. Therefore it is important to search for a numerical methods that share this feature. Much attention has been given to symplectic integrators for the numerical solution of Hamiltonian systems. However, it is well known that the local error of a non-symplectic method is smaller than that of the symplectic method, the error produce during the integration process is slower for the symplectic method. thus, for a large interval of integration of Hamiltonian systems the symplectic method will be more efficient than the non-symplectic method. A simple example to show how a symplectic method is propagated is by the use of an undamped harmonic oscillator. In this study, our main attention is on the systems of second order ODEs (1); which is a generalized representation of periodic problems. After changing the ODE (1) in to 
an equivalent system of first order ODEs, we get a Hamiltonian system. Beside the symplecticity, one can take in to account that the solutions of the ODE (1) have oscillatory character. In that case trigonometric fitting is a suitable choice. Motivated by the work of Simos in [7], we construct a symplectic third order four stage explicit trigonometrically-fitted RKN method. The remaining part of this paper is structured as follows: In Section 2 we give the basic theory of an explicit Runge-Kutta-Nyström method, Hamiltonian system, construction of Hamiltonian system and definition of a symplectic RKN method. Section 3 deals with the derivation of the proposed method. In Section 4 we analyze the algebraic order of the new method from its local truncation error. In Section 5 we present the numerical results and Section 6 deals with the conclusion.

\section{Basic Theory}

The general form of an explicit $m$-stage RKN method is given by:

$$
\begin{aligned}
y_{n+1} & =y_{n}+h y_{n}^{\prime}+h^{2} \sum_{i=1}^{m} b_{i} f\left(x_{n}+c_{i} h, Y_{i}\right), \\
y_{n+1}^{\prime} & =y_{n}^{\prime}+h \sum_{i=1}^{m} d_{i} f\left(x_{n}+c i h, Y_{i}\right), \\
Y_{i} & =y_{n}+c_{i} h y_{n}^{\prime}+h^{2} \sum_{j=1}^{i-1} a_{i j} f\left(x_{n}+c i h, Y_{i}\right) .
\end{aligned}
$$

or in Butcher Tableau as: where $a_{i j}, b_{j}, d_{j}$ and $c_{j}$ are the RKN parameters which

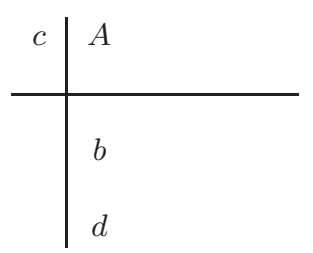

are assumed to be real and $m$ is the number of stages of the method. Introducing the $m$-dimensional vectors $c, b, d$ and $m \times m$ matrix A, where $c=\left[c_{1}, c_{2}, \ldots, c_{m}\right]^{T}$, $b=\left[b_{1}, b_{2}, \ldots b_{m}\right]^{T}, d=\left[d_{1}, d_{2}, \ldots d_{m}\right], A=\left[a_{i j}\right]$, respectively. 


\subsection{Hamiltonian Systems}

Hamiltonian systems are linear or nonlinear systems with particular symmetry that allows the stability of equilibrium points to be found and the solutions curves to be drawn even though actual solutions are not obtained. For these systems, the derivatives $\frac{d x}{d t}$ and $\frac{d y}{d t}$ are partial derivatives of a function called Hamiltonian function $(H(x, y))$.

$$
\begin{aligned}
& \frac{d x}{d t}=\frac{\partial H}{\partial y}, \\
& \frac{d y}{d t}=-\frac{\partial H}{\partial x} .
\end{aligned}
$$

Now, In terms of the variables of the systems we have: $\frac{\partial}{\partial x} \frac{d x}{d t}=-\frac{\partial}{\partial y} \frac{d y}{d t}$. Which enable us to write the system as:

$$
\begin{aligned}
& \frac{d x}{d t}=f(x, y), \\
& \frac{d y}{d t}=g(x, y) .
\end{aligned}
$$

Therefore, a system is Hamiltonian if: $\frac{\partial f}{\partial x}=-\frac{\partial g}{\partial y}$. In other words, a system that is Hamiltonian can be identified by this property. For example, The undamped harmonic oscillator $y^{\prime \prime}=-100 y$ is a linear Hamiltonian system. Changing this in to an equivalent system of first order ODE, we have:

$$
\begin{aligned}
& x^{\prime}=y=f(x, y), \\
& y^{\prime}=-100 x=g(x, y) .
\end{aligned}
$$

From above, we can see that: $\frac{\partial f}{\partial x}=\frac{d y}{d x}=0$ and $\frac{\partial g}{\partial y}=0$

Therefore, $\frac{\partial f}{\partial x}=-\frac{\partial g}{\partial y}$ implying that the undamped harmonic oscillator is a Hamiltonian system.

\subsection{Constructing Hamiltanion Systems}

The integral $\int \frac{\partial H}{\partial y} d y$ allows the Hamiltonian function to be determined up to a function that depends only on $x: H(x, y)=\int \frac{\partial H}{\partial y} d y+\Phi(x)=\int f(x, y) d y+\Phi(x)$, using the other system variable

$$
\frac{\partial H}{\partial x}=-g(x, y)
$$




$$
=\frac{\partial}{\partial x} \int f(x, y) d y+\frac{d}{d x} \Phi(x) .
$$

Rearranging we have:

$$
\frac{d}{d x} \Phi(x)=-g(x, y)-\frac{\partial}{\partial x} \int f(x, y) d y
$$

Integrating $f(x, y)$ with respect to $x$, we have:

$$
H(x, y)=\int f(x, y) d y+\Phi(x)
$$

Integrating both side with respect to $x$ of equation (6), we have:

$$
\Phi(x)=\int \frac{d}{d x} \Phi(x) d x+C
$$

Setting $C=0$ and substituting equation (8) in (7), we have the Hamiltonian.

Definition 1. A Runge-Kutta-Nyström method $(2)-(4)$ is said to be symplectic if it satisfies these two conditions:

$$
\begin{aligned}
b_{i} & =d_{i}\left(1-c_{i}\right), i=1,2, \ldots, m, \\
a_{i j} & =d_{j}\left(c_{i}-c_{j}\right), i, j=1,2, \ldots, m .
\end{aligned}
$$

or equivalently,

$$
\operatorname{det}\left[\begin{array}{cc}
\frac{\partial y_{n+1}}{\partial y_{n}} & \frac{\partial y_{n+1}}{\partial y_{n}^{\prime}} \\
\frac{\partial y_{n+1}^{\prime}}{\partial y_{n}} & \frac{\partial y_{n+1}^{\prime}}{\partial y_{n}}
\end{array}\right]=1
$$

\section{Construction of the New Method}

In this section, we will derive a four-stage third order symplectic explicit trigonometrically-fitted RKN method. In this work, a third-order four-stage with phase-lag order six as derived by Mohamad in [11] will be used. The coefficients of the method are given in Table 1 below:

When an explicit Runge-Kutta-Nyström method (2) - (4) is applied for $k=4$ to the test equation $y^{\prime \prime}=-w^{2} y$, the method become: 
Table 1: The SRKN4(3,6) Method [11]

\begin{tabular}{c|cccc}
0 & & & & \\
$\frac{1}{4}$ & 0.01735693733 & & & \\
$\frac{3}{4}$ & 0.05207081201 & 0.2201621183 & & \\
0.6015339728 & 0.04176314989 & 0.1547889282 & -0.09501810531 & \\
& & & & \\
& 0.06942774935 & 0.3302431775 & 0.1599997439 & -0.05967067075 \\
& 0.06942774935 & 0.4403242367 & 0.6399989756 & -0.1497509616
\end{tabular}

$y_{n+1}=y_{n}+h y_{n}^{\prime}+h^{2} \sum_{i=1}^{k} b_{i}\left(-w^{2} Y_{i}\right)$,

and

$$
y_{n+1}^{\prime}=y_{n}^{\prime}+h \sum_{i=1}^{k} d_{i}\left(-w^{2} Y_{i}\right) \text {. }
$$

where

$$
\begin{aligned}
& Y_{1}=y_{n}+c_{1} h y_{n}^{\prime}, \\
& Y_{2}=y_{n}+c_{2} h y_{n}^{\prime}-h^{2} a_{21} w^{2} Y_{1}, \\
& Y_{3}=y_{n}+c_{3} h y_{n}^{\prime}+h^{2}\left(-a_{31} w^{2} Y_{1}-a_{32} w^{2} Y_{2}\right), \\
& Y_{4}=y_{n}+c_{4} h y_{n}^{\prime}+h^{2}\left(-a_{41} w^{2} Y_{1}-a_{42} w^{2} Y_{2}-a_{43} w^{2} Y_{3}\right) .
\end{aligned}
$$

Let $y_{n}=e^{I w x}, y_{n}^{\prime}=I w e^{I w x}, y_{n+1}=e^{I w(x+h)}$ and $y_{n+1}^{\prime}=I w e^{I w(x+h)}$ and substituting in the equations $(11)-(16)$ and by using $e^{I v}=\cos (v)+I \sin (v)$ and comparing the real and imaginary part, we get the following system of equations:

$$
\begin{aligned}
& \cos (v)=1-v^{2} \sum_{i=1}^{4} b_{i}\left(1-v^{2} \sum_{j=1}^{3} a_{i j} Y_{j} e^{-I w x}\right) \\
& \sin (v)=v-v^{2} \sum_{i=1}^{4} b_{i} c_{i} v
\end{aligned}
$$




$$
\begin{aligned}
& \sin (v)=v \sum_{i=1}^{4} d_{i}\left(1-v^{2} \sum_{j=1}^{3} a_{i j} Y_{j} e^{-I w x}\right), \\
& \cos (v)=1-v^{2} \sum_{i=1}^{4} d_{i} c_{i} .
\end{aligned}
$$

where $v=w h$. Solving (17)-(20) using the coefficients of the method in Table 1 for $b_{2}, b_{3}, d_{2}, c_{3}$, we obtain the Taylor series expansion of the solution as given in (21).

$$
\begin{aligned}
b_{2}= & 0.3302474193-0.003269258848 v^{2}+0.0006624540319 v^{4}-0.00001232981110 v^{6} \\
& +0.00000001201292823 v^{8}-0.000000002415442190 v^{10}-3.347289386 \times 10^{-11} v^{12}+\ldots, \\
b_{3}= & 0.1599955019+0.004832120002 v^{2}-0.000009434253472 v^{4}-0.0000003408799608 v^{6} \\
& -0.000000007194753488 v^{8}-0.0000000001197562843 v^{10}-2.089039884 \times 10^{-12} v^{12} \\
& -2.101816634 \times 10^{-14} v^{14}+\ldots, \\
d_{2}= & 0.4403483712-0.000002031618000 v^{2}+0.002415798360 v^{4}-0.0001020990252 v^{6} \\
& +0.0000009836055400 v^{8}-0.000000007979728660 v^{10}+2.208678810 \times 10^{-11} v^{12} \\
& -3.813573763 \times 10^{-13} v^{14}-3.807738827 \times 10^{-15} v^{16}+\ldots, \\
c_{3}= & 0.7500132564-0.002441347370 v^{2}-0.00005172691604 v^{4}-0.00000002027291620 v^{6} \\
& +0.00000004591147348 v^{8}+0.0000000008759990916 v^{10}+2.877456882 \times 10^{-11} v^{12} \\
& +7.141345980 \times 10^{-13} v^{14}+\ldots
\end{aligned}
$$

This lead to the new method denoted as SETFRKN4(3,6).

\section{Algebraic Order and Error Analysis}

In this section, we perform local truncation error analysis, based on the Taylor series expansion of the actual solution $y\left(x_{n}+h\right)$, the first derivative of the actual solution $y^{\prime}\left(x_{n}+h\right)$, the approximate solution $y_{n+1}$, and the first derivative of the approximate solution $y_{n+1}^{\prime}$. The local truncation error (LTE) of $y$ and its first derivative $y^{\prime}$ is given below: 


$$
\begin{aligned}
L T E & =y_{n+1}-y\left(x_{n}+h\right), \\
L T E_{d e r} & =y_{n+1}^{\prime}-y^{\prime}\left(x_{n}+h\right) .
\end{aligned}
$$

The principal terms of the LTE for $y$ and $y^{\prime}$ of the new method SETFRKN4(3,6) is given in $(23)$, where it is shown that the order of the new method is three. In order to obtain the PLTE, we have to collect all the occurring partial derivatives of $f(x, y)$ from (1) with respect to $x$ and $y$.

$$
\begin{aligned}
\text { PLTE }= & -h^{4}\left(0.002858180003803371425 f_{x x}+0.005716360007606742850 y^{\prime} f_{x y}\right. \\
& +0.002858180003803371425\left(y^{\prime}\right)^{2} f_{y y}+0.001562861164628852337 f_{y} y^{\prime \prime} \\
& \left.+0.001562861210 w^{2} y^{\prime \prime}\right)+O\left(h^{5}\right), \\
P L T E_{d e r}= & \frac{h^{4}}{24}\left(f_{x x x}+3 y^{\prime} f_{y x x}+3 y^{\prime \prime} f_{x y}+3\left(y^{\prime}\right)^{2} f_{x y y}+3 y^{\prime} f_{y y} y^{\prime \prime}+\left(y^{\prime}\right)^{3} f_{y y y}+f_{y} f_{x}+\left(f_{y}\right)^{2} y^{\prime}\right) \\
& -h^{3}\left(0.00000275086007273243 f_{x x}+0.00000550172014546486 y^{\prime} f_{x y}\right. \\
& \left.+0.00000275086007273243\left(y^{\prime}\right)^{2} f_{y y}\right)-h^{3}\left(-0.00000203159802582186 f_{y} y^{\prime \prime}\right. \\
& \left.-0.000002031618000 w^{2} y^{\prime \prime}\right)+O\left(h^{5}\right) .
\end{aligned}
$$

\section{Problems Tested and Numerical Results}

In this section, we will apply the new method to some second-order ordinary differential equation problems. The following symplectic and non-symplectic RKN methods are used for the numerical comparisons.

- SETFRKN4(3,6): The new four-stage third order symplectic explicit trigonometrically-fitted RKN method derived in this paper,

- SRKN3(3,6): The three-stage third order symplectic explicit RKN method with phase-lag order six derived by Mohamad in [11],

- RKN4G: The fourth-order three-stage RKN method derived by Garcia in [14], and 


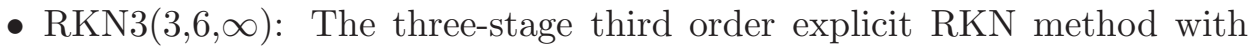
phase-lag order six and zero dissipative derived by Senu in [8].

Problem 1.(Harmonic Oscillator) Anastassi and Kosti in [12]

$$
y^{\prime \prime}=-100 y, y(0)=1, y^{\prime}(0)=2,
$$

The exact solution is

$$
y(x)=-\frac{1}{5} \sin (10 x)+\cos (10 x) .
$$

Problem 2. (Almost Periodic Problem) Mohamad in [11]

$$
\begin{aligned}
& y_{1}^{\prime \prime}=-y_{1}+0.001 \cos (x), y_{1}(0)=1, y_{1}^{\prime}(0)=0 \\
& y_{2}^{\prime \prime}=-y_{2}+0.001 \sin (x), y_{2}(0)=0, y_{2}^{\prime}(0)=0.9995
\end{aligned}
$$

The exact solution is

$$
\begin{aligned}
& y_{1}(x)=\cos (x)+0.0005 x \cos (x), \\
& y_{2}(x)=\sin (x)-0.0005 x \sin (x) .
\end{aligned}
$$

Problem 3. Anastassi and Kosti in [12]

$y^{\prime \prime}=-v^{2} y(x)+\left(v^{2}-1\right) \sin (x), y(0)=1, y^{\prime}(0)=v+1, x \geq 0$, where $v>>1$,

The exact solution is

$$
y(x)=\sin (10 x)+\cos (10 x)+\sin (x) .
$$

Problem 4. Senu in [8]

$$
y^{\prime \prime}=-y+x, y(0)=1, y^{\prime}(0)=2,
$$

The exact solution is

$$
y(x)=\cos (x)+\sin (x)+x .
$$

The accuracy strategy used is finding $\log _{10}$ of the maximum global error,

$$
M A X E R R=\log _{10} \max \left\|y\left(x_{n}\right)-y_{n}\right\|,
$$

where $x_{n}=x_{0}+n h, n=1,2,3, \ldots\left(T-x_{0}\right) / h$. In this paper, we denote $T$ as the interval used for the integration.

The numerical results are shown in Tables 2-5.

We also display the accuracy of these methods graphically in figures 1 to 4 . 
Table 2: Numerical results for problem 1

\begin{tabular}{|c|c|c|c|c|}
\hline $\mathbf{h}$ & Methods & $\mathrm{T}=1000$ & $\mathrm{~T}=5000$ & $T=10000$ \\
\hline \multirow[t]{4}{*}{0.025} & SETFRKN4 $(3,6)$ & $6.000147(-7)$ & $3.000695(-6)$ & $6.127334(-6)$ \\
\hline & $\operatorname{SRKN} 3(3,6)$ & $2.909168(-4)$ & $2.909168(-4)$ & $3.3 .289169(-4)$ \\
\hline & RKN4G & $6.205026(-2)$ & $3.052327(-1)$ & $5.935375(-1)$ \\
\hline & $\operatorname{RKN} 3(3,6, \infty)$ & $2.909152(-4)$ & $2.909152(-4)$ & $3.435898(-4)$ \\
\hline \multirow[t]{4}{*}{0.05} & SETFRKN4 $(3,6)$ & $1.223357(-6)$ & $6.088788(-6)$ & $1.225812(-5)$ \\
\hline & $\operatorname{SRKN} 3(3,6)$ & $2.348434(-3)$ & $1.823382(-2)$ & $3.874725(-2)$ \\
\hline & RKN4G & $8.664679(-1)$ & $1.540438(+0)$ & $1.540438(+0)$ \\
\hline & $\operatorname{RKN} 3(3,6, \infty)$ & $2.348432(-3)$ & $1.824115(-2)$ & $3.876189(-2)$ \\
\hline \multirow[t]{4}{*}{0.075} & SETFRKN4 $(3,6)$ & $1.902483(-6)$ & $9.551457(-6)$ & $1.902038(-5)$ \\
\hline & $\operatorname{SRKN3}(3,6)$ & $4.110906(-2)$ & $2.372272(-1)$ & $4.791851(-1)$ \\
\hline & RKN4G & $1.391214(+0)$ & $1.391214(+0)$ & $1.391214(+0)$ \\
\hline & $\operatorname{RKN} 3(3,6, \infty)$ & $4.111048(-2)$ & $2.372344(-1)$ & $4.791997(-1)$ \\
\hline \multirow[t]{4}{*}{0.1} & SETFRKN4 $(3,6)$ & $2.662733(-6)$ & $1.333361(-5)$ & $2.653967(-5)$ \\
\hline & $\operatorname{SRKN3}(3,6)$ & $2.752468(-1)$ & $1.339798(+0)$ & $2.020189(+0)$ \\
\hline & RKN4G & $1.279301(+0)$ & $1.279301(+0)$ & $1.279301(+0)$ \\
\hline & $\operatorname{RKN} 3(3,6, \infty)$ & $2.752481(-1)$ & $1.339805(+0)$ & $2.020196(+0)$ \\
\hline
\end{tabular}

Table 3: Numerical results for problem 2

\begin{tabular}{|c|c|c|c|c|}
\hline $\mathbf{h}$ & Methods & $T=1000$ & $\mathrm{~T}=5000$ & $\mathrm{~T}=10000$ \\
\hline \multirow[t]{4}{*}{0.025} & SETFRKN4 $(3,6)$ & $2.184220(-8)$ & $1.202666(-7)$ & $1.882064(-7)$ \\
\hline & $\operatorname{SRKN} 3(3,6)$ & $4.549746(-7)$ & $1.674375(-6)$ & $4.578916(-6)$ \\
\hline & RKN4G & $6.268906(-7)$ & $4.866342(-6)$ & $1.613212(-5)$ \\
\hline & $\operatorname{RKN} 3(3,6, \infty)$ & $3.221998(-7)$ & $7.468672(-7)$ & $1.142362(-6)$ \\
\hline \multirow[t]{4}{*}{0.05} & SETFRKN4 $(3,6)$ & $1.317210(-7)$ & $7.035181(-7)$ & $1.391787(-6)$ \\
\hline & $\operatorname{SRKN} 3(3,6)$ & $2.569510(-6)$ & $5.142489(-6)$ & $1.068136(-5)$ \\
\hline & RKN4G & $1.004116(-5)$ & $7.811003(-5)$ & $2.627705(-4)$ \\
\hline & $\operatorname{RKN} 3(3,6, \infty)$ & $2.581249(-6)$ & $6.114042(-6)$ & $1.144586(-5)$ \\
\hline \multirow[t]{4}{*}{0.075} & SETFRKN4 $(3,6)$ & $4.202444(-7)$ & $2.115351(-6)$ & $4.368256(-6)$ \\
\hline & $\operatorname{SRKN} 3(3,6)$ & $8.320939(-6)$ & $1.445156(-5)$ & $2.668259(-5)$ \\
\hline & RKN4G & $5.084022(-5)$ & $3.953611(-4)$ & $1.330306(-3)$ \\
\hline & $\operatorname{RKN} 3(3,6, \infty)$ & $8.703650(-6)$ & $2.051119(-5)$ & $3.877658(-5)$ \\
\hline \multirow[t]{4}{*}{0.1} & SETFRKN4 $(3,6)$ & $9.754692(-7)$ & $4.909562(-6)$ & $9.942111(-6)$ \\
\hline & SRKN3(3,6) & $1.953365(-5)$ & $3.263652(-5)$ & $5.745523(-5)$ \\
\hline & RKN4G & $1.606798(-4)$ & $1.249630(-3)$ & $4.205253(-3)$ \\
\hline & $\operatorname{RKN} 3(3,6, \infty)$ & $2.062338(-5)$ & $4.856175(-5)$ & $9.155271(-5)$ \\
\hline
\end{tabular}


Table 4: Numerical results for problem 3

\begin{tabular}{|c|c|c|c|c|}
\hline $\mathbf{h}$ & Methods & $T=1000$ & $\mathrm{~T}=5000$ & $T=10000$ \\
\hline \multirow[t]{4}{*}{0.025} & SETFRKN4 $(3,6)$ & $8.602342(-7)$ & $4.186587(-6)$ & $8.523618(-6)$ \\
\hline & $\operatorname{SRKN} 3(3,6)$ & $3.020171(-4)$ & $3.020171(-4)$ & $6.655309(-4)$ \\
\hline & RKN4G & $8.609385(-2)$ & $4.233520(-1)$ & $8.230410(-1)$ \\
\hline & $\operatorname{RKN} 3(3,6, \infty)$ & $3.021801(-4)$ & $3.062383(-4)$ & $6.863074(-4)$ \\
\hline \multirow[t]{4}{*}{0.05} & SETFRKN4 $(3,6)$ & $2.206600(-6)$ & $8.930309(-6)$ & $1.746995(-5)$ \\
\hline & $\operatorname{SRKN} 3(3,6)$ & $4.293087(-3)$ & $2.676248(-2)$ & $5.522888(-2)$ \\
\hline & RKN4G & $1.201341(+0)$ & $1.201341(+0)$ & $1.201341(+0)$ \\
\hline & $\operatorname{RKN} 3(3,6, \infty)$ & $4.297201(-3)$ & $2.677425(-2)$ & $5.524999(-2)$ \\
\hline \multirow[t]{4}{*}{0.075} & SETFRKN4 $(3,6)$ & $5.404536(-6)$ & $1.592486(-5)$ & $2.902879(-5)$ \\
\hline & $\operatorname{SRKN} 3(3,6)$ & $6.228579(-2)$ & $3.347018(-1)$ & $6.711267(-1)$ \\
\hline & RKN4G & $1.929449(+0)$ & $1.929449(+0)$ & $1.929449(+0)$ \\
\hline & $\operatorname{RKN} 3(3,6, \infty)$ & $6.229966(-2)$ & $3.347039(-1)$ & $6.711440(-1)$ \\
\hline \multirow[t]{4}{*}{0.1} & SETFRKN4 $(3,6)$ & $1.300358(-5)$ & $2.743251(-5)$ & $4.570371(-5)$ \\
\hline & $\operatorname{SRKN} 3(3,6)$ & $3.960430(-1)$ & $1.879627(+0)$ & $2.824299(+0)$ \\
\hline & RKN4G & $1.773207(+0)$ & $1.773207(+0)$ & $1.773207(+0)$ \\
\hline & $\operatorname{RKN} 3(3,6, \infty)$ & $3.960249(-1)$ & $1.879651(+0)$ & $2.824317(+0)$ \\
\hline
\end{tabular}

Table 5: Numerical results for problem 4

\begin{tabular}{|c|c|c|c|c|}
\hline $\mathbf{h}$ & Methods & $\mathrm{T}=1000$ & $\mathrm{~T}=5000$ & $\mathrm{~T}=10000$ \\
\hline \multirow[t]{4}{*}{0.025} & SETFRKN4 $(3,6)$ & $1.027990(-8)$ & $5.504171(-8)$ & $9.120413(-8)$ \\
\hline & $\operatorname{SRKN3}(3,6)$ & $4.647316(-7)$ & $1.277958(-6)$ & $2.228004(-6)$ \\
\hline & RKN4G & $8.611927(-7)$ & $4.310487(-6)$ & $8.524657(-6)$ \\
\hline & $\operatorname{RKN} 3(3,6, \infty)$ & $2.921239(-7)$ & $3.027080(-7)$ & $3.027080(-7)$ \\
\hline \multirow[t]{4}{*}{0.05} & SETFRKN4 $(3,6)$ & $1.771605(-8)$ & $9.499581(-8)$ & $1.760764(-7)$ \\
\hline & SRKN3(3,6) & $2.494562(-6)$ & $3.180916(-6)$ & $4.096439(-6)$ \\
\hline & RKN4G & $1.379246(-5)$ & $6.903953(-5)$ & $1.380617(-4)$ \\
\hline & $\operatorname{RKN} 3(3,6, \infty)$ & $2.344371(-6)$ & $2.351930(-6)$ & $2.362362(-6)$ \\
\hline \multirow[t]{4}{*}{0.075} & SETFRKN4 $(3,6)$ & $2.574836(-8)$ & $1.266999(-7)$ & $2.577654(-7)$ \\
\hline & $\operatorname{SRKN3}(3,6)$ & $8.080317(-6)$ & $8.668262(-6)$ & $9.468105(-6)$ \\
\hline & RKN4G & $6.981822(-5)$ & $3.493498(-4)$ & $6.991754(-4)$ \\
\hline & $\operatorname{RKN} 3(3,6, \infty)$ & $7.939240(-6)$ & $7.939240(-6)$ & $7.939240(-6)$ \\
\hline \multirow[t]{4}{*}{0.1} & SETFRKN4 $(3,6)$ & $3.370155(-8)$ & $1.1 .684839(-7)$ & $3.415953(-7)$ \\
\hline & $\operatorname{SRKN3}(3,6)$ & $1.900296(-5)$ & $1.948996(-5)$ & $2.013470(-5)$ \\
\hline & RKN4G & $2.207326(-4)$ & $1.104077(-3)$ & $2.209373(-3)$ \\
\hline & $\operatorname{RKN} 3(3,6, \infty)$ & $1.888495(-5)$ & $1.888495(-5)$ & $1.888495(-5)$ \\
\hline
\end{tabular}




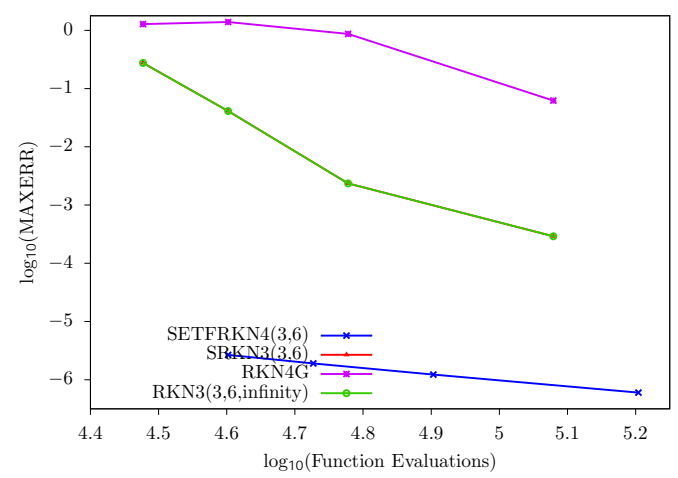

Figure 1: The efficiency curve for Problem 1 with $t_{\text {end }}=1000$ and $h=i(0.025), i=1,2,3,4$.

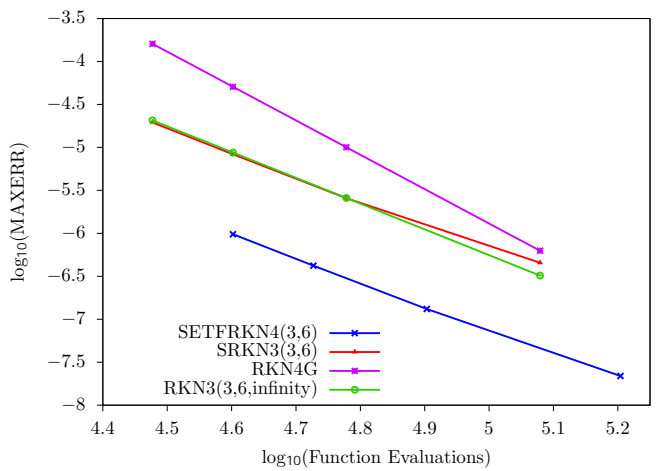

Figure 2: The efficiency curve for Problem 2 with $t_{\text {end }}=1000$ and $h=i(0.025), i=1,2,3,4$.

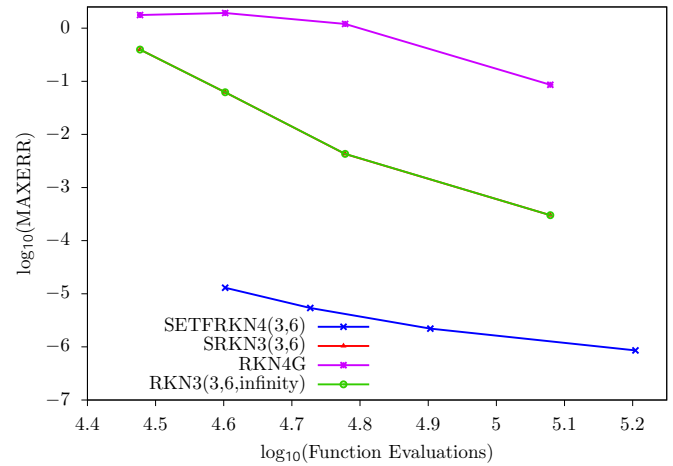

Figure 3: The efficiency curve for Problem 3 with $t_{\text {end }}=1000$ and $h=i(0.025), i=1,2,3,4$. 


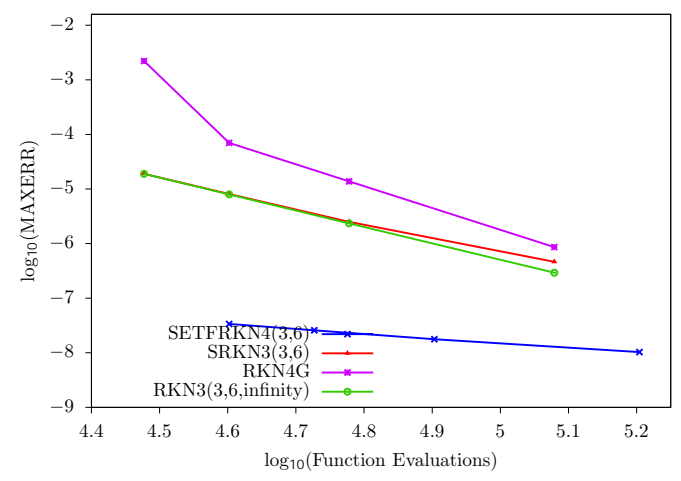

Figure 4: The efficiency curve for Problem 4 with $t_{\text {end }}=1000$ and $h=i(0.025), i=1,2,3,4$.

\section{Conclusion}

In this study, we have derived a four-stage third order symplectic explicit trigonometrically-fitted RKN method for the solutions of oscillatory initial value problems. The numerical results of the new method are compared with the other existing non-symplectic and symplectic RKN methods. The global error of the new method is smaller than the other existing non-symplectic and symplectic RKN methods. Hence, the new method is more efficient.

\section{Acknowledgments}

We are thankful to the referees for their constructive comments and the Institute of Mathematical Research (INSPEM), the Department of Mathematics, Universiti Putra Malaysia for the support and assistance during the research work. 


\section{References}

[1] H. Van de Vyver, "A symplectic exponentially fitted modified Runge-Kutta-Nyström method for the numerical integration of orbital problems ",New Astronomy, 10 (2005), 261-269.

[2] H. Van de Vyver, "A fourth-order symplectic exponentially-fitted integrator", Computer Physics Communications, 174 (2006), 255-262.

[3] H. Van de Vyver, "'A Symplectic Runge-Kutta-Nyström method with minimal phaselag", Physics Letters A, 260 (2014), 482-493.

[4] A. Tocino, and J. Vigo-Aguiar, "Symplectic condition for exponential fitting RungeKutta-Nyström methods", Mathematical and Computer Modelling, 42 (2005), 873-876.

[5] Z. Kalogiratou, T. Monovasilis and T. E. Simos, "A fourth order modified trigonometrically-fitted symplectic Runge-Kutta-Nyström method ", Computer Physics Communications, 185 (2014), 3151-3155.

[6] J. Franco, and I. Gomez "Symplectic explicit methods of Runge-Kutta-Nyström type for solving pertubed oscillators, Journal of Computational and Applied Mathematics, 260 (2014), 482-493.

[7] T. E. Simos, "An exponentially-fitted Runge-Kutta method for the numerical integration of initial-value problems with periodic or oscillating solutions, Computer Physics Communications, 115 (1998), 1-8.

[8] N. Senu, "Runge-Kutta Nyström methods for solving oscillatory problems", Ph.D diss., Department of Mathematics, Faculty of Science, 43400 UPM Serdang, Malaysia, 2009.

[9] J. C. Butcher, "Numerical methods for ordinary differential equations", Wiley \& Sons LTD., England, 2008.

[10] Xinyuan Wu, Xiong You and Bin Wang, "Structure preserving algorithms for oscillatory differential equations", Science Press Beijing \& Springer-Verlag Berlin Heidelberg, Beijing, 2013.

[11] M. Mohamad, "Explicit Runge-Kutta Nyström methods with high order dispersion and dissipation for solving oscillatory second order ordinary differential equation", M.sc diss., Department of Mathematics, Faculty of Science, 43400 UPM Serdang, Malaysia, 2013.

[12] Z. Anastassi and A. Kosti, "A 6(4) optimized embedded Runge-Kutta Nyström pair for the numerical solution of periodic problems ", Journal of Computational Applied Mathematics, 275 (2013), 311-320.

[13] N. Senu, M. Suleiman, F. Ismail and M. Othman, "A singly diagonally implicit RungeKutta-Nyström method for solving oscillatory problems", IAENG International Journal of Applied Mathematics, 41 (2011), 155-161.

[14] A. Garcia, P. Martin, and A. B. Gonzalez et al, "New methods for oscillatory problems based on classical codes", Applied Numerical Mathematics, 42 (2002), 141-157.

[15] N. Senu, M. Suleiman, and F. Ismail, "An embedded explicit Runge-Kutta-Nyström method for solving oscillatory problems", Physica Scripta, 80 (2009), 015005.

[16] D. F. Papadopoulos, Z. Anastassi, A. Zacharias and T. E. Simos, "A phase-fitted RungeKutta-Nyström method for the numerical solution of initial value problems with oscillating solutions", Computer Physics Communications, 180 (2009), 1839-1846. 